ArtefaCToS. Revista de estudios de la ciencia y la tecnología

eISSN: $1989-3612$

Vol. 9, No. 2 (2020), 2. ${ }^{\text {E }}$ Época, 27-49

DOI: https://doi.org/10.14201/art2020922749

\title{
La comunicación pública de la ciencia y tecnología como acción política en un escenario de crisis
}

\section{Public Communication of Science and Technology as Political Ac- tion in a Crisis Scenario}

\section{Lucía CÉSPEDES*; Antonio CHIAVASSA FERREYRA**}

${ }^{*}$ Centro de Investigaciones y Estudios en Cultura y Sociedad (CIECS), Universidad Nacional de Córdoba (UNC)/Consejo Nacional de Investigaciones Científicas y Técnicas (CONICET), Argentina

lucicespedes27@gmail.com

${ }^{* *}$ Facultad de Ciencias de la Comunicación, Universidad Nacional de Córdoba, Argentina

antoniochiavassaferreyra@gmail.com

Recibido: 05/02/2020. Revisado: 20/04/2020. Aceptado: 21/10/2020

\section{Resumen}

En este trabajo abordamos los aspectos comunicacionales del proceso de movilización del sector científico argentino en contra de las políticas de ajuste implementadas por el gobierno nacional en el período 2015-2019, atendiendo a cómo los científicos implementan estrategias de comunicación pública de la ciencia y la tecnología (CPCT). Buscamos caracterizar la relación entre la CPCT y la política en y para la ciencia, ya que es una de las principales formas en que los agentes del campo científico se vuelcan a la esfera pública para disputar y legitimar sus intereses. Para esto, reseñamos las acciones de lucha llevadas a cabo por trabajadores del CONICET en 2018-2019. A partir del análisis de los géneros discursivos de documentos públicos dirigidos a las autoridades nacionales y a la ciudadanía argentina, identificamos dimensiones como los actores interpelados, los modelos de ciencia y CPCT subyacentes, y las representaciones transmitidas. También nos interrogamos sobre el rol de la CPCT en un contexto regional inestable donde se alternan modelos políticos y económicos con agendas científicas y tecnológicas dispares. Finalizamos reflexionando acerca del objeto posible de la CPCT en un sistema tecnocientífico desarticulado y en crisis. 
Palabras clave: CONICET; esfera pública; acción política; política científica; géneros discursivos.

\begin{abstract}
In this paper we approach the communicational aspects of the social mobilization process by the Argentinian scientific sector against the austerity policies implemented by the national government in the 2015-2019 period, attending to the strategies of public communication of science and technology (PCST) deployed by scientists involved in the conflict. We seek to characterize the relationship between science policy, the politics of science, and PCST, since this is one of the main ways in which agents of the scientific field turn to the public sphere to dispute and legitimize their interests. In order to do so, we review collective actions carried out by CONICET workers in 2018-2019. By analyzing the speech genres in public documents addressed to national authorities and citizens, we identify actors involved, underlying models of science and PCST, and social representations transmitted. We also question the role of PCST in an unstable regional context where political-economical models with very dissimilar agendas are systematically alternated. We conclude by reflecting on the possible object of PCST in a technoscientific system which is disarticulated and undergoing a crisis.
\end{abstract}

Keywords: CONICET; public sphere; political action; scientific policy; speech genres.

\title{
1. Introducción
}

En este trabajo tenemos dos intenciones. En primer lugar, describir las estrategias de comunicación pública de la ciencia y la tecnología (en adelante, $\mathrm{CPCT}^{1}$ ) implementadas por un sector de la comunidad científica argentina para exhibir en la esfera pública los problemas de financiación y los resultados de las "políticas de austeridad" sobre el sistema científico y tecnológico nacional durante la

\footnotetext{
${ }^{1}$ La categoría de "comunicación pública de la ciencia y la tecnología" remite a una elección metodológica que no se condice del todo con nuestra postura epistemológica. Junto con Alcíbar $(2009,167)$, consideramos que la distinción entre ciencia (básica) y tecnología ya no se sostiene: "no representan compartimentos estancos, sino dominios fuertemente imbricados", por lo que gran parte de la investigación actual es de un carácter netamente tecnocientífico. Sin embargo, al interior del campo científico, y muy fuertemente en la Argentina, aún se los concibe como términos que se suelen vincular pero que se siguen viendo como ámbitos separados. Como ejemplo, baste señalar que, al 2018, sólo 3,11\% de los investigadores de CONICET pertenecían al área de conocimiento Tecnología. En el campo de los estudios de la comunicación de la ciencia esta noción está en discusión. A los fines de este trabajo, y con el objetivo de acercarnos a lo que observamos como la idea más extendida en el discurso de los agentes analizados, mantenemos los términos "ciencia y tecnología" por separado.
} 
gestión presidencial de Mauricio Macri. En segundo lugar, interrogarnos sobre las posibilidades y potencialidades de la CPCT en un contexto regional inestable donde se alternan modelos políticos y económicos con agendas científicas y tecnológicas dispares. Comprender la magnitud de estos problemas exige reconocer la necesaria relación entre la política y la CPCT, ya que es una de las principales formas en que los agentes del campo científico (Bourdieu, 1994) se vuelcan a la esfera pública para disputar sus intereses.

Las prácticas de comunicación de la ciencia, en este sentido, se han expandido y recorren un camino de institucionalización (Cortassa y Rosen, 2019), a la par de evolucionar desde el tradicional modelo de déficit cognitivo hasta los actuales planteos que apuntan al diálogo entre saberes (Alcíbar, 2015). Sin embargo, si bien la práctica de comunicar contenidos científicos a un público no especializado cuenta con amplios antecedentes, estos temas aún no son jerarquizados en las agendas mediáticas de la misma forma que, por ejemplo, la política o la economía.

En el escenario de los países latinoamericanos, y en la Argentina en particular, esta disputa por instalar la CPCT discurre a la par de un conflicto por sostener, afianzar y expandir la ciencia y la tecnología en sí misma. Como observamos en otros trabajos, el desarrollo de la comunicación pública de la ciencia y la tecnología en la Argentina "ha ido de la mano de la evolución e institucionalización de la investigación científica en el país, la cual a su vez se ha visto fuertemente marcada por la orientación de las políticas estatales de CyT en cada momento histórico" (Céspedes, 2019, 283). En un patrón consistente con el caso de muchos países periféricos, las instituciones de investigación y desarrollo (I+D) argentinas son frágiles. La inestabilidad resultante de la puja entre modelos disímiles y en ocasiones irreconciliables condiciona la consolidación y continuidad del sistema científico-tecnológico (Hurtado, 2019). Durante el gobierno de Mauricio Macri (2015-2019), la retracción del sector se hizo evidente. La desjerarquización del Ministerio de Ciencia, Tecnología e Innovación Productiva, la gradual pero sostenida reducción de los fondos estipulados en el presupuesto anual y el recorte cuantitativo en la convocatoria anual para ingreso a la carrera del investigador científico en el Consejo Nacional de Investigaciones Científicas y Técnicas (CONICET), son algunos de los ejemplos más evidentes (volveremos a esto más adelante).

Coincidimos en que es imposible desagregar la CPCT de las políticas públicas orientadas hacia el sector. En parte la relación es directa. Un gran porcentaje de las acciones de comunicación se generan por iniciativas de instituciones públicas vinculadas a la investigación y al desarrollo, o financiadas por agentes del Estado. Pero también hay una relación indirecta, aunque evidente. Si se reduce sistemáticamente la producción científica y tecnológica de un país por falta de políticas públicas, ¿`cuál es el objeto de la comunicación pública de la ciencia y la tecnología? 
Por supuesto, las políticas públicas adversas a la ciencia, la tecnología, y su comunicación pública no se aplican sin resistencias. En los últimos años, se multiplicaron las protestas de investigadores, estudiantes y profesionales en contra de lo que ellos consideran un desmantelamiento del sistema científico y tecnológico nacional. Además, diversos agentes externos al ámbito de producción de conocimiento se hicieron eco de las exigencias y denuncias de los representantes del sistema científico. No cabe duda de que estas acciones de protesta son de índole política: el objetivo es instalar una demanda en la esfera pública. En tanto intervención política, los agentes del campo científico buscan legitimar su posición ante la sociedad civil y construir alianzas para impulsar sus intereses.

En este contexto, la tradicional (y cada vez más discutida) autonomía del campo científico se tambalea. Ante la necesidad de instalar y legitimar socialmente un reclamo que excede a lo sectorial (pues todo conflicto del campo científico-tecnológico involucra nociones más amplias de desarrollo, soberanía, dependencia, y modelos de país), los agentes movilizados despliegan una serie de estrategias comunicacionales entre las que detectamos elementos del ámbito de la CPCT. Al decir de Alcíbar $(2015,3)$, esta es una "noción paraguas" útil para referir a "todas aquellas actividades con las que la cultura científica se incorpora a la cultura popular”. En esto reside la particularidad del asunto: la CPCT, ya sea realizada por científicos o comunicadores, siempre transmite representaciones acerca de la ciencia (Hilgartner, 1990). En otras palabras, la CPCT es un recurso para instalar representaciones que justifiquen las demandas de la comunidad científica ante la retracción del sistema científico tecnológico.

Con estas consideraciones en mente, en el primer apartado de este artículo daremos algunas precisiones conceptuales que orientarán el trabajo, describiremos nuestras decisiones metodológicas al recortar nuestro objeto de análisis, y relataremos brevemente el contexto sociopolítico en que se desarrollaron los conflictos abordados. En el segundo apartado analizamos los aspectos políticos y comunicacionales de los choques entre el gobierno nacional y el sector científico argentino durante los años 2018 y 2019. Procuraremos mostrar cómo, en momentos de crisis del sector, se enfatiza el aspecto político de la CPCT, pero el contenido científico tradicional (de corte más "divulgativo") no deja de estar presente. Como veremos, los científicos argentinos han tenido iniciativas muy variadas a tal fin: algunas disruptivas como intervenciones, performances, o ferias de ciencias en la vía pública; otras más tradicionales como la publicación de comunicados, artículos, o entrevistas en los medios masivos; el uso de redes sociales para viralizar consignas en forma de hashtags; hasta acciones más típicamente relacionadas con el reclamo social como marchas o tomas de edificios públicos. Conviven distintas acciones y géneros de enunciación en el arsenal comunicacional de la comunidad científica; la CPCT es solo una de las modalidades para hacer pública la demanda, pero sin duda es una de las más originales y específicas. En el tercer apartado analizaremos, desde la perspectiva bajtiniana de géneros discursivos, algunos rasgos de los documentos publicados por los representantes 
de CONICET que diagnostican y denuncian la situación de crítica del sistema científico y tecnológico. Entre los innumerables aspectos con potencialidad de ser analizados, tomaremos dos: cómo se presenta el sector científico y tecnológico en tanto enunciador, y cuáles son los enunciatarios a los que se apela, tanto para informar como para comprometer con su demanda. Finalmente, en la discusión y conclusiones sintetizamos los puntos principales del artículo y sugerimos algunas posibles formas de pensar constructivamente en el potencial de la CPCT como forma de intervención política en el espacio público, pero también como objeto de política(s) en sí.

\section{Aspectos contextuales, conceptuales y metodológicos}

No es el objetivo de este trabajo ahondar en detalles públicamente conocidos sobre los recortes a la CyT argentina en los últimos ańos, pero se hace necesario brindar algunos datos para contextualizar el clima en el que se desarrollan los procesos de lucha que sí buscamos analizar.

El ajuste acusado en la Argentina sobre el sector científico-tecnológico se enmarca en una tendencia regional común. En el año 2014, el crecimiento sostenido de los recursos destinados a $\mathrm{I}+\mathrm{D}$ (en millones de dólares internacionales en PPC) se estancó en Iberoamérica, América Latina y el Caribe, mientras que en 2015 decreció por primera vez desde el año 2000. En ningún otro bloque regional del mundo se registró esta tendencia negativa (RICyT, 2018). Particularmente, en la Argentina la retracción del sector coincide con el cambio de signo político en el gobierno nacional a partir de 2015, lo cual trajo aparejado un cambio en el modelo económico, social y productivo del país. Demostrando una vez más la vigencia del modelo del "péndulo" postulado hace décadas por el economista Marcelo Diamand (1983), el proyecto político de Cambiemos se construyó como antagónico y refundacional respecto del gobierno anterior. Esto se tradujo en la aplicación rápida y sucesiva de políticas de corte neoliberal clásico, tendientes a la reducción de las capacidades y esferas de acción estatales, la toma de deuda externa, la apuesta por las inversiones de capital extranjero, la devaluación del peso, y la desindustrialización de la base productiva del país.

Cabe aclarar que el sistema científico argentino está compuesto por múltiples instituciones que financian, promueven y ejecutan investigación científica, la mayoría de las cuales pertenecen al ámbito del Estado nacional. Entre las más relevantes, se pueden mencionar el Consejo Nacional de Investigaciones Científicas y Técnicas (CONICET), el Instituto Nacional de Tecnología Industrial (INTI), el Instituto Nacional de Tecnología Agropecuaria (INTA), la Comisión Nacional de Energía Atómica (CNEA), y la Comisión Nacional de Actividades Aeroespaciales (CONAE). Asimismo, las universidades nacionales constituyen importantes polos de investigación y formación de recursos humanos altamente calificados. Los vaivenes en política científica afectan a cada institución de forma 
diferente, e históricamente cada una ha respondido a los cambios pendulares de modelos políticos y económicos en función de sus características particulares y de su propia cultura institucional. Por lo tanto, entendemos que la noción de "comunidad científica" es una sobresimplificación, operativa y útil sin duda a la hora de remitir rápidamente al conjunto de agentes e instituciones involucrados en la producción, circulación y apropiación del conocimiento científico-tecnológico, pero que incluye una amplia gama de intereses, reivindicaciones y necesidades particulares.

Ante esta diversidad, y debido a las modestas pretensiones del presente trabajo, es necesario hacer un recorte si queremos profundizar en un sector de esta comunidad y evitar generalizaciones infundadas. Para nuestro estudio decidimos enfocarnos en las acciones y discursos públicos del personal científico de CONICET, ya que puede argüirse que es la institución científica dominante en la Argentina. Al 2018, empleaba 10619 investigadores y 10895 becarios (doctorales y posdoctorales) distribuidos en 298 institutos de investigación en todas las provincias del país. Asimismo, en ese año contabilizaba 2636 personal de apoyo a la investigación y 1380 empleados administrativos. Lejos están esas cifras, de, por ejemplo, los 3595 profesionales, 1313 técnicos, 2035 personal de apoyo, y 321 becarios que el INTA empleaba a diciembre de 2017 (Nocetti, Palioff y Della Torre, 2018).

Más allá de su volumen, históricamente el CONICET se ha constituido en el imaginario social y del propio campo como el paradigma de lo que la investigación científica es y aspira a ser en la Argentina. Por lo tanto, sus criterios y esquemas de apreciación tienden a imponerse en el campo. Para Kreimer (2011), desde su fundación (muy ligada al capital científico, social y simbólico de su primer presidente, Bernardo Houssay) el CONICET fue determinante:

en el establecimiento de criterios para la evaluación de las actividades, y para cristalizar la existencia de una élite internacionalizada que tuviera la legitimidad de un hablar legítimo 'en nombre de la ciencia'. Así, podemos afirmar que hacia los años sesenta esta institución había logrado introducir más o menos los mismos criterios que imperaban por entonces en los países centrales. Y esta tendencia se va a mantener, prácticamente, hasta la actualidad. Kreimer $(2011,68)$

Esta importancia y visibilidad es un arma de doble filo para la institución. Por un lado, es una voz legítima y autorizada para, justamente, pronunciarse públicamente "en nombre de la ciencia" y usar su poder de presión en favor de todo el sector CyT nacional. Por otro, tal visibilidad lleva, en cierta medida, a una identificación reduccionista "ciencia argentina = CONICET". Al modo de una estrella cuyo brillo no permite apreciar a otras de menor intensidad, la dimensión del CONICET hace necesario un esfuerzo consciente por discernir los reclamos comunes a todo el sector científico-tecnológico y las necesidades particulares de esta institución, que pueden o no coincidir con la situación de otros institutos 
y centros de investigación argentinos. Así, la desproporción entre instituciones conlleva una desigual capacidad de impulsar demandas políticas e instalarlas en la opinión pública. Esta heterogeneidad se reduce al reclamo de un sector específico que asume un rol protagónico y no siempre atiende a las necesidades particulares de los demás centros de producción de $\mathrm{I}+\mathrm{D}$.

$\mathrm{Al}$ mismo tiempo, el volumen del CONICET lo hizo, en los últimos años, blanco fácil del discurso de "racionalización" sostenido por una gestión cuyas acciones apuntaron al achicamiento del Estado. Esto se aprecia no sólo en las acciones concretas que afectaron al CONICET (sin dejar de considerar que otras instituciones de menores recursos y menor capacidad de lobby han sido aún más perjudicadas), sino en la discusión pública que se desató alrededor de la conducción, rumbo y objetivos de la institución. En efecto, el CONICET enfrentó una sistemática campaña de desprestigio mediática en la que participaron desde anónimos usuarios de Twitter ensañados especialmente con las investigaciones del área de Ciencias Sociales y Humanidades, hasta su expresidente, el físico Alejandro Cecatto, quien lo calificó como una institución "inviable" al poco tiempo de asumir. Teniendo en cuenta que ninguna política se puede aplicar en el vacío discursivo, y que la CPCT no sólo comunica contenido científico sino también representaciones y valoraciones sobre esta práctica (Hilgartner, 1990), sería interesante en futuros trabajos caracterizar las modalidades de CPCT sostenidas desde el gobierno macrista en torno al conflicto del sector.

Por estas razones, es decir, el peso material y simbólico de CONICET tanto en el campo científico argentino como en la esfera pública, el análisis a partir de ahora se centrará en las intervenciones de sus integrantes alrededor del conflicto sectorial. Quedará para otros trabajos un seguimiento de las consecuencias del ajuste sobre las demás instituciones que comprenden el sistema de CyT argentino y las estrategias adoptadas por sus miembros.

\section{La CPCT en defensa del sistema científico-tecnológico nacional (2018- 2019)}

Para hacer una breve reseña de la relación espinosa entre el gobierno macrista y el sector científico argentino, podemos identificar como primer hecho altamente conflictivo entre el CONICET y esa gestión gubernamental nacional la reducción de los ingresos a la Carrera de Investigador Científico (CIC) en la convocatoria 2016, cuando las plazas para incorporar investigadores pasaron a ser sólo 385, en comparación con las 943 ofrecidas en 2015. Esto motivó la toma pacífica del Polo Científico Tecnológico ubicado en Ciudad Autónoma de Buenos Aires y de Centros Científico Tecnológicos (CCT) en todo el país, así como movilizaciones e intervenciones en la vía pública bajo consignas (inevita- 
blemente devenidas hashtags ${ }^{2}$ ) como "Investigar es trabajar", "No a los despidos encubiertos", y la ubicua "No al ajuste en Ciencia y Tecnología”. La magnitud de las medidas de lucha se resolvió parcialmente con la extensión de las becas a aquellos que habían obtenido doble recomendación para su ingreso a CIC, pero habían quedado afuera, así como la promesa de reubicar a esos investigadores en otras instituciones científicas y universidades nacionales. A partir de entonces, ciertos sectores de la comunidad científica argentina mantuvieron, con vaivenes en el volumen de participación y frecuencia de reunión, una actividad asamblearia en estado de "alerta permanente".

En los meses siguientes se recrudecieron las medidas de retracción de las instituciones científicas del país; una de las más significativas sería la disolución, en 2018, del Ministerio de Ciencia, Tecnología e Innovación Productiva y su transformación en una Secretaría de Gobierno subsumida en el Ministerio de Educación, Cultura, Ciencia y Tecnología ${ }^{3}$. El deterioro progresivo de las condiciones salariales y de trabajo motivaron el involucramiento de cada vez más integrantes del campo (y agentes externos) en las actividades de protesta. En diciembre de 2018, la publicación de una carta abierta dirigida al "Presidente de la Argentina Mauricio Macri y las autoridades argentinas en ciencia y tecnología” marcó un clivaje en el proceso de lucha y movilización que hasta el momento venía siendo sostenido por la organización de becarios doctorales, posdoctorales, e investigadores jóvenes: más de 1200 destacados investigadores de diferentes países y filiaciones institucionales firmaron mostrando su adhesión, entre ellos, 11 científicos laureados con el Premio Nobel en Física, Química, o Medicina.

Ya en 2019, la conmemoración del Día del Investigador Científico en la Argentina, el 10 de abril ${ }^{4}$, fue el marco de, nuevamente, movilizaciones y eventos en espacios públicos de múltiples ciudades, bajo consignas como "Más ciencia, menos dependencia" o "Ciencia es soberanía". Ese mismo año, durante la Semana de Mayo, unidades ejecutoras y centros científico-tecnológicos pertenecientes a CONICET realizaron sesiones de debate y conferencias de prensa denominadas "Cabildos Abiertos". Las protestas de abril y mayo fueron las expresiones políticas de mayor relevancia durante el año; luego la atención mediática y la expectativa de la comunidad científica (y de la sociedad argentina en su conjunto) fueron capturadas por las elecciones presidenciales.

\footnotetext{
${ }^{2}$ La estrategia de lucha y visibilización del conflicto en redes sociales discurrió a la par de las acciones en la vía pública y los comunicados que son objeto de este trabajo. El despliegue de formas de protesta y CPCT en redes en defensa de la ciencia involucra múltiples dimensiones comunicativas, políticas y semióticas, y merece un análisis en sí mismo, pero excede los alcances del presente artículo.

${ }^{3}$ En diciembre de 2019, al asumir la gestión de Alberto Fernández, se lo restituyó al nivel de Ministerio de Ciencia, Tecnología e Innovación.

${ }^{4}$ Celebrado en conmemoración del nacimiento del primer Premio Nobel en ciencias argentino y latinoamericano, el doctor Bernardo Houssay, en 1887.
} 


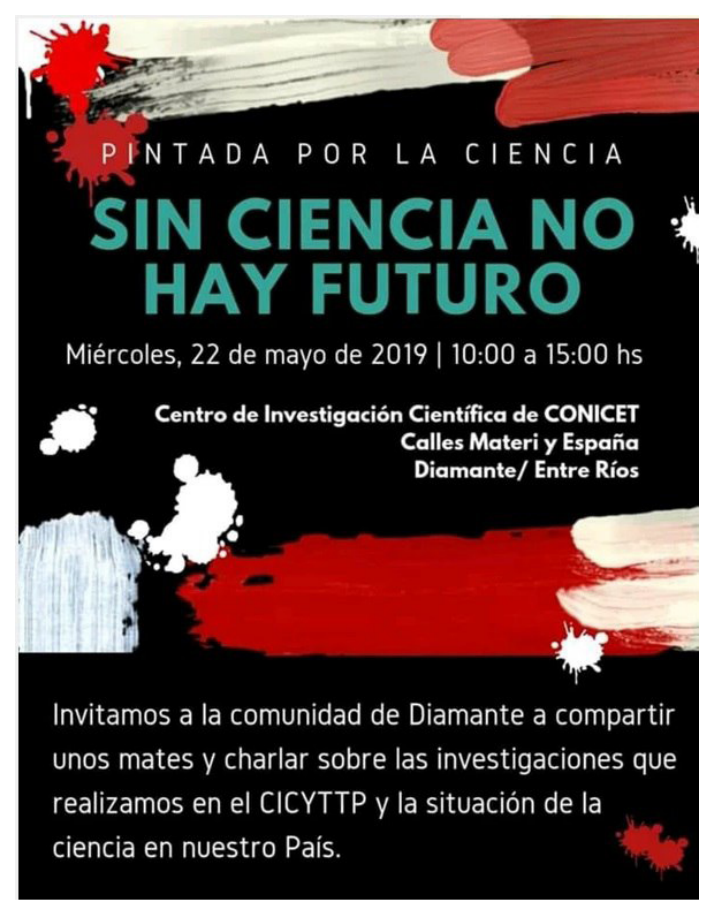

Imagen 1. Volante invitando a las actividades del Cabildo Abierto realizado en la ciudad de Diamante, Entre Ríos. Fuente: Blog de la Mesa Coordinadora del Plenario Nacional, https://cienciaenargentina.wixsite.com/mesacoordinadora

El repertorio de manifestaciones políticas de los trabajadores y trabajadoras del sistema científico tecnológico argentino ciertamente incluyó estrategias de protesta social tradicionales, como movilizaciones en vías públicas u ocupación de edificios. Sin embargo, estas acciones se matizaron con exposiciones diversas orientadas a divulgar el conocimiento producido y su relevancia. Persistió una particularidad en sus modalidades de protesta: a la par de sus demandas, los científicos realizaron intervenciones comunicativas para justificarlas.

En efecto, a diferencia de otros sectores, los defensores del sistema de CyT deben legitimar el impacto social del trabajo científico que, en general, tiene efectos diferidos y a largo plazo. En consecuencia, se necesitan acciones comunicativas que transmitan conceptos y prácticas de elevada complejidad. En este escenario, la CPCT es una herramienta estratégica para fortalecer el reclamo, ya que divulgar prácticas y resultados de investigaciones junto con algunos conceptos generales de las disciplinas científicas sirve de fundamento a la demanda política de inversión en el sistema de CyT.

Por supuesto, los agentes del campo científico argentino seleccionaron algunas de las acciones posibles dentro de la diversidad de la CPCT. En particular, tuvieron preponderancia aquellas que implicaron un despliegue en la vía pública, que interpelan a los transeúntes y que pueden atraer la atención de los medios. Una característica recurrente de las manifestaciones de los científicos en la esfera 
pública fueron las actividades didácticas y de divulgación de la investigación. El formato recurrente fue el de la "feria de ciencias", que es una forma de exhibición usual en la CPCT y una de las modalidades más antiguas de presentación social de la ciencia. La comunicación interpersonal, fundada en la oralidad y los gestos de un expositor, son un rasgo constitutivo de esta modalidad de CPCT. Se asimila a la presentación de un orador para un auditorio, pero sin la distancia que impone un escenario. Así, el acto de comunicación interpersonal se impone sobre la impersonalidad de la tarima. Por otro lado, la feria de ciencia apela al recurso de la curiosidad que siempre despierta la CyT.

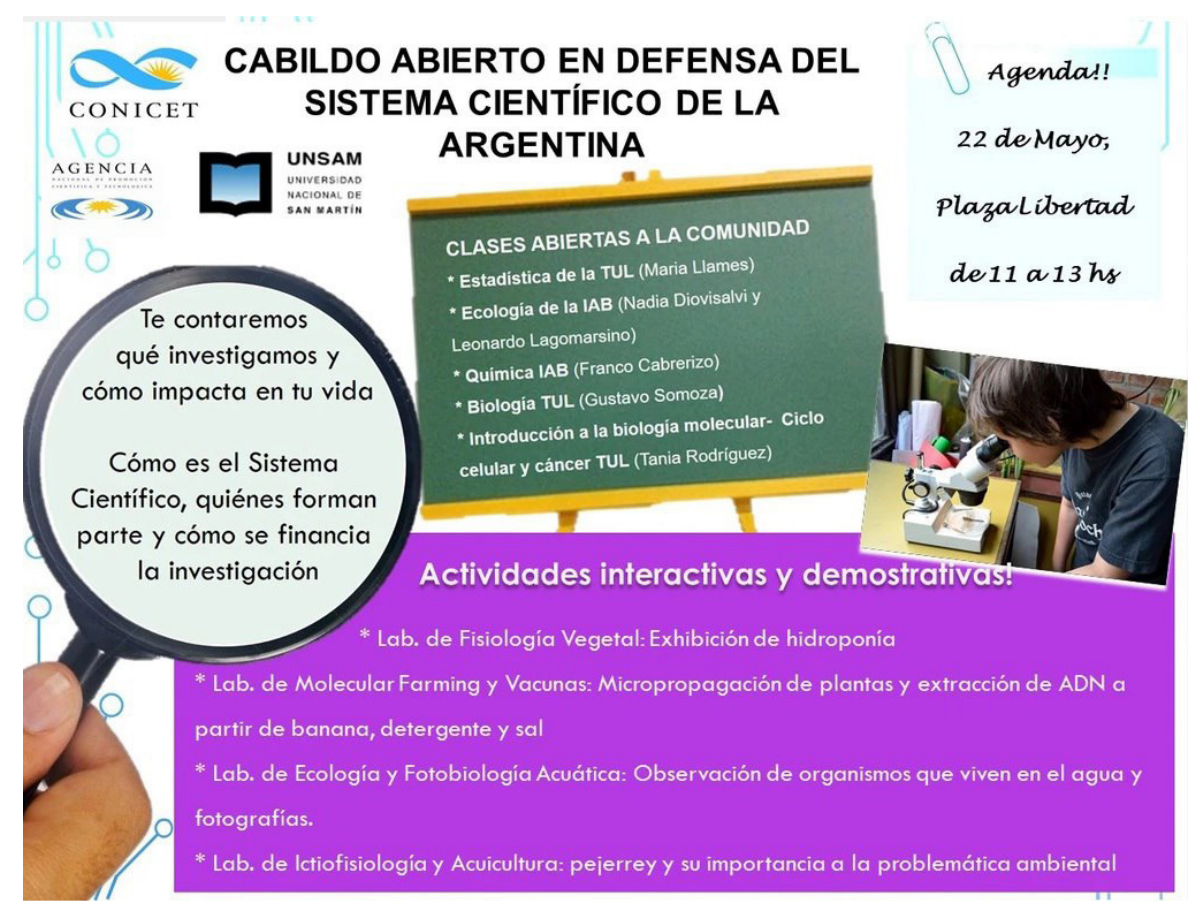

Imagen 2. Volante invitando a las actividades del Cabildo Abierto realizado en la Universidad Nacional de San Martín, Buenos Aires. Fuente: Blog de la Mesa Coordinadora del Plenario Nacional, https://cienciaenargentina.wixsite.com/mesacoordinadora

Las manifestaciones de abril y mayo se realizaron en la Ciudad Autónoma de Buenos Aires y en al menos 18 provincias, y si bien cada uno de estos nodos organizó las jornadas de manera independiente, hubo un alto nivel de coordinación en la determinación de las fechas, rango horario de las actividades, y consignas centrales del reclamo. Esto permitió mostrar la presencia y alcance de los centros de investigación vinculados al CONICET a lo largo y ancho del país, visibilizando la presencia de científicos fuera de los centros académicos de mayor peso y ligados a las universidades nacionales de mayor tradición, como pueden ser Buenos Aires, Córdoba, o La Plata. La presencia de CPCT fue variable; muchos grupos priorizaron formas tradicionales de expresión política. Sin 
embargo, stands con atracciones científicas y charlas sobre las investigaciones del CONICET coexistieron con las movilizaciones en la vía pública y frente a los edificios de ciencia.

Además, se destaca la elaboración de consignas de gran carga simbólica para transmitir de manera gráfica la situación por la que atravesaba el sector científico. Bajo el lema "La ciencia con la soga al cuello", los manifestantes marcharon con sogas de ahorcado para simbolizar la asfixia del sistema científico; en "La ciencia está de luto" los manifestantes cargaron un ataúd para escenificar el funeral de la ciencia argentina. Este recurso creativo no es exclusivo de esta coyuntura; desde la década de los ' 90 , los científicos argentinos se han apropiado del concepto de "lavar los platos" para llevar adelante sus reclamos en la vía pública en contextos de recorte presupuestario e indiferencia política hacia el sector CyT. Desde entonces, no ha sido infrecuente la imagen de grupos de investigadores instalando ferias de ciencias en plazas o parques con fuentes donde se pudiera realizar la mímica del lavado de platos.

El sitio de instalación de las ferias de ciencias o de realización de las protestas no fue casual: se priorizaron espacios nodales en cada ciudad, de alta circulación de público, y en horarios que permitieran la cobertura en vivo por parte de los matutinos radiales y televisivos de mayor audiencia. En este sentido, en algunas ciudades argentinas el emplazamiento de las instalaciones emblemáticas de CONICET coincide con lugares céntricos y concurridos, por lo que una manifestación a sus puertas tendría una eficiencia simbólica doble: la ocupación de un espacio público de gran visibilidad sumada a la rápida identificación de los sujetos movilizados como miembros de la comunidad científica. Por otra parte, en aquellas ciudades donde los edificios de CONICET se encuentran apartados o no están integrados en los circuitos urbanos más concurridos, se optó por la realización de las actividades de protesta y comunicación en plazas o calles que son típicamente escenarios de manifestaciones sociales de todo tipo.

Recordemos que la esfera pública incluye los espacios comunes y se amplía a los circuitos informativos de los medios masivos. En ese sentido, las acciones de CPCT en la vía pública tienen la virtud de atraer la atención de los medios por la noticiabilidad, ya que son sucesos fuera de lo ordinario. En efecto, las acciones de denuncia que no involucran un elemento de CPCT ocupan un lugar en la agenda mediática resultante de su relevancia social, pero carece del atractivo de lo extraordinario. En consecuencia, la cobertura mediática de las ferias y perfomances difunde por circuitos exógenos (Verón, 1998) la demanda política, la representación que la comunidad científica desea transmitir de sí misma y el contenido estricto de divulgación.

\footnotetext{
${ }^{5}$ En alusión a la frase proferida en 1994 por el entonces Ministro de Economía Domingo Cavallo, quien, ante las advertencias de la socióloga y demógrafa Susana Torrado acerca de los efectos perjudiciales de la política científica aplicada por el gobierno menemista, manifestó que la investigadora debería dedicarse "a lavar los platos".
} 
La mayoría de las actividades hasta aquí descritas han sido históricamente organizadas y llevadas a cabo por aquellos agentes que ocupan posiciones dominadas dentro del campo científico-académico: becarios doctorales y posdoctorales, e investigadores jóvenes. Esto es consistente con las observaciones de Kreimer, Levin y Jensen (2011), quienes encontraron que las actividades de CPCT se distribuyen de manera heterogénea entre los investigadores de CONICET en función de su edad y, principalmente, en función de su prestigio, autoridad y grado de consagración. Los autores consideran que las propias actividades de CPCT son valoradas diferencialmente al interior del campo científico; en consecuencia, señalan que los investigadores senior tienden a, por ejemplo, dar entrevistas a los medios masivos, mientras que los investigadores junior son más activos en actividades consideradas de menor prestigio, como jornadas de puertas abiertas, ferias, o charlas escolares.

En efecto, en el proceso de lucha que venimos reseñando, los investigadores jóvenes han tenido una marcada presencia en el contexto de manifestaciones en el espacio público o en roles de representantes sindicales. A través de la aparición de este colectivo en móviles televisivos, radiales, o en la prensa gráfica o web, la figura del becario se recortó, por un lado, como uno de los principales motorizadores y conductores del reclamo sectorial general; por otro lado, como un agente con características particulares dentro del campo científico, especialmente en lo que concierne a su cualidad de científico en formación y a su estatus laboral ambiguo. Asimismo, la voluntad de instalar el reclamo en la agenda de los medios masivos requirió que becarios, técnicos, administrativos e investigadores de CONICET recurrieran aún a otros géneros discursivos, en este caso relacionados con lo periodístico: la entrevista y la elaboración de gacetillas de prensa propias.

Cabe destacar que los agentes con posiciones inferiores en la jerarquía del campo científico son los primeros afectados por la retracción del sistema científico tecnológico. Doctorandos y jóvenes investigadores, aún en vías de acumular capital científico, son los más susceptibles a la expulsión del campo debido a la reducción de sus dimensiones. Consecuentemente, deben adecuar sus estrategias para garantizar su continuidad, perseverando dentro de las instituciones que los cobijan u optando por la inserción en centros de investigación extranjeros. En general, no disponen de reconocimiento como figuras destacadas de la ciencia y deben aglutinarse en un colectivo para visibilizar sus reclamos. Por otro lado, son proclives a interpelar aliados externos al campo científico para motorizar sus demandas, en parte debido a la ausencia de una posición que los inmunice de los efectos de la retracción y la falta de líneas de diálogo directas con tomadores de decisiones y demás actores de poder.

Al contrario, los agentes con suficiente capital, aunque no son inmunes, son capaces de morigerar las situaciones económicas adversas porque disponen de estabilidad institucional. Por ende, tienen menos inclinación a manifestar reclamos políticos públicos en defensa del sector científico tecnológico. Además, una 
posición elevada en el campo científico-académico habilita el contacto directo con representantes de las esferas del poder político y económico, por lo que cabe pensar en estrategias de protesta y resistencia diferenciadas de acuerdo al capital social y administrativo poseído y, por ende, la posición ocupada en el campo. Una evidencia de esto es la relativamente tardía participación explícita de directores de institutos en contra de la retracción del sistema científico tecnológico.

En abril de 2019, un grupo de directores y directoras de unidades ejecutoras (UEs) y CCTs de CONICET se autoconvocaron en la ciudad de Córdoba en un Plenario Nacional a fin de intercambiar información, debatir sobre la situación del sector, y elaborar una propuesta para superar la crisis y un plan de actividades para impulsar estos reclamos. Como corolario de dos jornadas de trabajo en comisiones y reuniones plenarias generales (que contaron con la participación y exposiciones de la situación específica de cada estamento del personal de CONICET), se generó un sitio web ${ }^{6}$ donde se publicaron para su difusión una serie de textos que detallan las condiciones materiales del sector de CyT y de CONICET en especial, las reivindicaciones salariales y presupuestarias del sector, y sus reclamos políticos más amplios. Tanto la reunión como estos textos tuvieron amplia cobertura y difusión mediática. En el siguiente apartado nos ocupamos del análisis de dichos comunicados.

\section{Análisis de discurso de los comunicados}

Las estrategias para defender el sistema científico tecnológico abrevan de una multiplicidad de tácticas discursivas y textuales para complementar la acción colectiva directa. En esa línea, el análisis de las actividades y los enunciados de CPCT sería inconsistente si no consideramos estos otros textos que articulan las demandas más netamente políticas del sector. El despliegue de enunciados de diversa índole conforma una constelación de textos orientados a la misma finalidad en donde la CPCT es solo una parte del conjunto, aunque fundamental. Por supuesto, los rasgos genéricos de cada tipo de enunciado a veces son difíciles de amalgamar en un agregado uniforme y existen tensiones. Pero señalar esta complejidad es imposible sin un repaso de algunos textos relevantes.

Los documentos publicados por el Plenario de Directores pertenecen a un género discursivo que, desde una perspectiva acorde a Bajtin ${ }^{7}$, responde a determinada área de la praxis humana: la acción política en la esfera pública. Son textos

\footnotetext{
${ }^{6}$ Directoras y Directores de Institutos de CONICET - Blog de la Mesa Coordinadora del Plenario Nacional, https://cienciaenargentina.wixsite.com/mesacoordinadora

7 "El uso de la lengua se lleva a cabo en forma de enunciados (orales y escritos) concretos y singulares que pertenecen a los participantes de una u otra esfera de la praxis humana. [...] Cada enunciado separado, es por supuesto, individual pero cada esfera de uso de la lengua elabora sus tipos relativamente estables de enunciados, a los que denominamos géneros discursivos" (Bajtin, 2008, 245).
} 
que no pertenecen a los géneros recurrentes que se asocian al campo científico, cuyos enunciados característicos son los artículos de investigación publicados en revistas especializadas y sometidas a revisión por pares evaluadores, que son parte del conjunto de elementos que afirman la autonomía del campo. Es decir, las competencias necesarias para interpretar la especificidad de un texto científico exigen un nivel de inserción formal e informal a este ámbito. Sin embargo, los documentos de denuncia se inscriben en otro género, porque responden a otra esfera de la praxis humana. En ese sentido, si los científicos deben abandonar sus géneros usuales y recurrir a un tipo de enunciado asociado a otras prácticas, ¿cómo logran presentarse como científicos en la esfera pública? La CPCT parece ser el género discursivo suplementario que respalda la identidad de los científicos.

El primer documento público dado a conocer por el Plenario Nacional de Directores y Directoras de Institutos de CONICET se titula "Manifiesto de Córdoba 2019" (en adelante, "Manifiesto"). Recibió la adhesión de 244 autoridades representantes de 238 institutos ubicados en 22 provincias de la Argentina. En diciembre de 2018 CONICET contabilizaba 294 institutos; de esto resulta que un $81 \%$ apoyó la realización del Plenario y la redacción del Manifiesto. Este consiste en un breve pronunciamiento de una carilla de extensión, que se complementa y amplía con el documento "Llamamiento a la ciudadanía: ¡salvemos la ciencia argentina!" (en adelante, "Llamamiento"), también redactado y difundido en el marco del Plenario. Es un texto más largo y detallado, donde se dedican seis páginas a exponer la situación del sector CyT argentino y de CONICET en particular, así como las reivindicaciones y medidas concretas que se demandan. Incluye un apéndice de veinte tablas y gráficos elaborados en base a datos oficiales y públicos que dan cuenta del deterioro presupuestario y salarial desde 2015 . Finalmente, el documento titulado "A la ciudadanía y al conjunto de las fuerzas políticas argentinas: ¡sin ciencia no hay futuro!" (en adelante, "Sin ciencia”) fue consensuado por la Mesa Coordinadora del Plenario de Directoras y Directores de Institutos del CONICET para ser leído en las jornadas de Cabildo Abierto desarrolladas en todo el país el 22 de mayo de 2019.

En estos textos, hay una alternancia en los modos en que el enunciador se autoidentifica y presenta. Las referencias al sector CyT en tercera persona en el Llamamiento ("El sistema científico y tecnológico argentino está hoy en riesgo de desaparición...") y la primera persona en plural de los firmantes ("Como responsables de la dirección de Institutos del CONICET nos vemos en la obligación de denunciar...") conviven con un nosotros inclusivo ("Debemos ser claros, si queremos la supervivencia del sistema científico...") que comprende a la totalidad de la ciudadanía. Alternativamente, se manifiesta la crisis del sector con datos objetivos externos al enunciador y se invita a reflexionar en calidad de ciudadanos sobre la gravedad de la situación. Esta manera de presentar la voz de los agentes denunciantes se acompaña con el señalamiento de los valores con los que 
el sector de CyT pretende ser asociado. Estos elementos en conjunto constituyen el retrato del enunciador de la comunidad científica y tecnológica como actor colectivo.

En relación a estos valores, es llamativo que el Manifiesto y el Llamamiento lleven como encabezado una fotografía del monumento del Centenario de la Reforma, inaugurado en junio de 2018 en la Ciudad Universitaria de la Universidad Nacional de Córdoba como parte de la celebración de los cien ańos de la Reforma Universitaria de 1918. Se trata de una escultura de hierro basada en la histórica foto de ocho estudiantes sobre el techo del viejo Rectorado de la UNC, izando un trozo de cortina como bandera. No es la primera vez que, en procesos de conflicto y movilización, el sector científico-tecnológico argentino apela al imaginario de la Reforma del ' 18 . Por caso, el proceso de modernización de las universidades iniciado con el cese de la intervención y restitución de la autonomía universitaria luego del golpe militar de 1955 adoptaría esta misma denominación, reformismo. Si bien con el correr de los años, especialmente durante la década de 1960, serían cuestionados por los sectores más radicalizados de la academia y, sobre todo, del movimiento estudiantil, los reformistas de aquel momento trazaban una genealogía que conectaba sus iniciativas y reclamos con la histórica gesta de 1918. En el plano político, la garantía del gobierno tripartito de las universidades representaba un avance hacia un cogobierno más democrático, la atención a la función social de la universidad, y la ampliación de las posibilidades de acceso a la educación superior por parte de los sectores sociales más desfavorecidos. En el plano académico, se apuntaba a fortalecer los vínculos entre docencia e investigación y orientar la ciencia a las necesidades del desarrollo nacional (Feld, 2019). De esta manera, la construcción de una identidad como sector científico-académico se apuntala en los hitos y las conquistas de la Reforma y se establece una continuidad en esa línea de pensamiento y acción.

Por otro lado, en sus primeras líneas, los documentos analizados definen al desarrollo científico y tecnológico como condición necesaria para el avance del país en múltiples aspectos (económico, industrial, cultural) y para el bienestar general de la población. Esta afirmación, a priori reminiscente de un modelo lineal de innovación ${ }^{8}$, es complejizada más adelante a medida que se desagregan las consecuencias puntuales de las medidas gubernamentales sobre el CONICET. Cabe pensar entonces en que ese primer fragmento actúa como una estrategia retórica

\footnotetext{
${ }^{8}$ El modelo lineal de innovación usualmente aparece como el sustrato de la política científica implícita (Herrera, 2015 [1975]) ante la indefinición de lineamientos para el sector que articulen agentes y etapas de investigación con arreglo a ciertos fines. Bajo esta perspectiva, ciencia básica-ciencia aplicada-innovación tecnológica-apropiación y uso son etapas encadenadas secuencialmente. La acumulación de conocimiento es condición necesaria y suficiente para que los efectos de la ciencia se "derramen" en el mercado y se traduzcan en bienestar social. Esta fase implica la intervención de agentes que concreticen los resultados de la investigación científica en bienes o servicios pasibles de ser aprovechados por el resto de la sociedad mediante su colocación en el mercado y posterior comercialización. Siguiendo a Kreimer $(2006,210)$, "este modelo tuvo
} 
dirigida a establecer una base argumental y discursiva común entre enunciador y enunciatario acerca de la relevancia social de la CyT. Una afirmación general, amplia, directa, que es presentada como indiscutible ("pocos gobernantes se atreven a cuestionar públicamente la validez de esta afirmación”), sirve como punto de inicio para una discusión más detallada sobre el estado del sector.

Los lugares comunes acentuados en los documentos se apoyan en la doxa sobre la CyT extendida en el discurso social. En la representación colectiva y de sentido común, la CyT tiene un vínculo estrecho con el desarrollo económico y el crecimiento de la calidad de vida, en particular, las ciencias biomédicas, que tienen un impacto evidente y cuentan con una larga tradición de investigación en la Argentina. Como dijimos, destacar este repertorio de representaciones al inicio del texto es parte de una estrategia argumental. Si el papel de la CyT en el desarrollo y el bienestar es tan incuestionable, ¿por qué la falta de incentivos? Sin embargo, no es solo un artificio retórico, sino la apelación a un reconocimiento extendido en la sociedad que es parte de una estrategia de identificación y presentación del enunciador. La relación de la ciencia y la tecnología con el bienestar social es señalada como uno de los justificativos para promover el apoyo al sistema científico y tecnológico. Es decir, discontinuar las políticas públicas en el sector atenta contra la totalidad de la sociedad. En general, existe una enfática referencia, por parte de los científicos, al valor social de la investigación.

El "Manifiesto" es un documento corto con dos enunciatarios posibles. Primero, la sociedad argentina en general: "...nos vemos en la obligación de denunciar ante la sociedad...”. Esta construcción es interesante pues da cuenta de la magnitud de la crisis tal como es percibida por los agentes que la viven y la sufren en su actividad científica. Agotados los canales habituales, los directores y directoras recurren a una estrategia que usualmente no emplean ${ }^{9}$, es decir, la apelación al espacio público. Asimismo, plantea cierta dimensión ética de la denuncia: la situación del sector CyT es tal que estarían faltando a su deber, incumpliendo su obligación, si no la expusieran a la población. En segundo lugar, hay un enunciatario implícito a quien se dirige la demanda concreta de "un plan de salvataje de CONICET": el gobierno nacional. Si bien no se lo menciona explícitamente, queda claro que los cuatro puntos exigidos caen dentro de la esfera de incumbencia del Estado nacional: aumento de emergencia del presupuesto de CONICET, prórroga de becas de aquellos jóvenes científicos afectados por los recortes en el cupo para ingreso a CIC, la normalización institucional del Directorio de CONICET, y la restitución del Ministerio de Ciencia, Tecnología e Innovación

\footnotetext{
mayores consecuencias simbólicas que materiales: la mayor parte de los conocimientos producidos bajo esta lógica sirvió más para aumentar la visibilidad de los investigadores locales que para generar conocimiento localmente útil y apropiable".

${ }_{9}^{9}$ Como mencionamos anteriormente, las estrategias de reclamo varían en función del capital y posición en el campo científico-académico. Las acciones de protesta más directa son generalmente organizadas y conducidas por agentes de menor capital y posiciones subordinadas.
} 
Productiva. Es interesante señalar cómo el significante salvar está muy presente en todos los documentos. Incluso se manifestó gráficamente en las piezas y afiches confeccionados para la convocatoria a las marchas e intervenciones en el espacio público. Por ejemplo, como actividad para el Cabildo Abierto, el Centro Austral de Investigaciones Científicas (CADIC-CONICET), sito en la ciudad de Ushuaia, diseñó un logotipo donde un salvavidas rodea a un barco hundiéndose. La invitación era a fotografiarse con ese marco y compartir la imagen en redes sociales usando los hashtags \#22M y \#SalvemosLaCiencia.

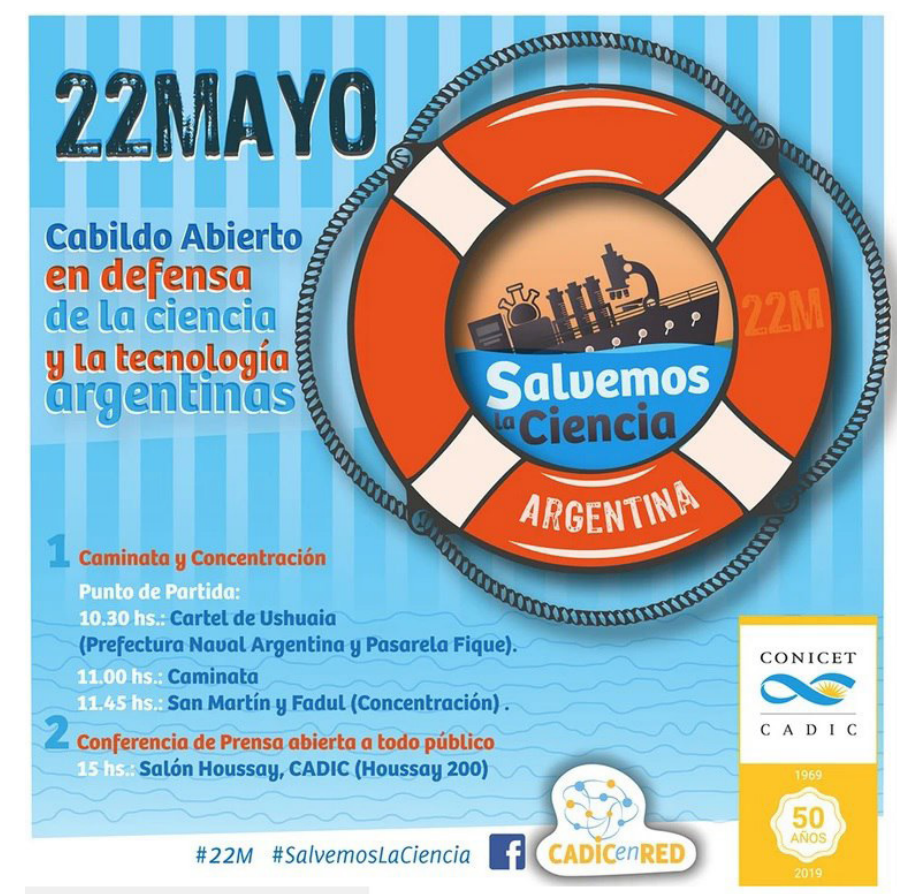

Imagen 3. Volante invitando a las actividades del Cabildo Abierto realizado en la ciudad de Ushuaia, Tierra del Fuego. Fuente: Blog de la Mesa Coordinadora del Plenario Nacional, https://cienciaenargentina.wixsite.com/mesacoordinadora

El "Llamamiento" presenta, nuevamente, dos enunciatarios; sin embargo, en su título queda claro cuál es el principal: el llamamiento a salvar la ciencia argentina es a la ciudadanía. El objetivo en relación a este enunciatario es alertar y convocar "a movilizarse para reclamar un cambio en las políticas aplicadas en el sector científico y tecnológico". El segundo enunciatario al que apela el Llamamiento no es ya exclusivamente el gobierno nacional de aquel momento, sino el conjunto de las fuerzas políticas argentinas. Éstas son convocadas "a pronunciarse en defensa de la ciencia y tecnología argentinas y a comprometerse en la ejecución de las medidas (...) que constituyen un programa de emergencia destinado a salvar nuestra base científica y tecnológica". El "Sin ciencia" coloca a ambos agentes, la ciudadanía y las fuerzas políticas del país, al mismo nivel y con una mayor preponderancia: el escrito los menciona expresamente en el título. 
La interpelación a la ciudadanía y a las fuerzas políticas eleva el nivel de compromiso que se exige al destinatario. Mientras que el Manifiesto se limita a denunciar ante la sociedad la situación crítica del sistema de CyT, el Llamamiento y el Sin ciencia pretenden involucrar activamente a sus enunciatarios. En el caso de las fuerzas políticas, se sigue con naturalidad su implicación debido a su papel de decisores en términos de política pública: "solicitamos a las fuerzas políticas no solamente un compromiso a largo plazo con el crecimiento del sistema científico nacional sino también el apoyo explícito a una serie de medidas de emergencia para salvar al sistema científico". Esto incluye a la fuerza que detentaba el poder gubernamental como al amplio espectro de actores políticos que aspiraban a ocupar la dirección del Estado. En relación a las primeras, las demandas son a corto plazo, más que nada medidas de emergencia que permitan, al menos, el normal funcionamiento de CONICET. Por otro lado, las reivindicaciones más generales y de mediano o largo plazo, que apuntan a revertir el ajuste sufrido por el sector, se dirigen de manera genérica y sin identificación partidaria a todos aquellos agentes políticos con posibilidad de ser partícipes del diseño e implementación de políticas de estado luego de la coyuntura electoral de octubre de 2019.

\section{Discusión y conclusiones}

El recorrido por el reciente conflicto entre el personal de CONICET y el anterior gobierno nacional argentino demuestra que las estrategias de CPCT con fines de resistencia política se usan para defender los sentidos ya previamente instalados en torno a la CyT. Precisamente, dichos sentidos circulan mediante la implementación de una CPCT enfocada en constituir determinadas representaciones sobre el campo científico en el espacio público, más que el mero contenido de divulgación. Este movimiento de apertura, de "presentarse en sociedad" por parte de agentes insertos en el campo CyT, necesariamente vuelve sobre el mismo campo y contribuye a discutir y reforzar una identidad compartida en tanto científicos comprometidos con la comunicación. Ambos aspectos, la CPCT como estrategia de resistencia y de constitución, son complementarios y en muchos casos simultáneos, pero en función del contexto sociopolítico se refuerza uno o el otro.

Los momentos de crisis en el sistema científico-tecnológico son cuando más se pone en evidencia la flaqueza de intentar sostener una visión dicotómica internalista-externalista para analizar los procesos del campo. La política irrumpe como una fuerza pretendidamente externa, pero durante estos procesos queda de manifiesto cómo es un componente intrínseco de la actividad científico-tecnológica. En etapas de ciencia normal, o de movimiento pendular hacia modelos de gobierno que apoyen al sector, esto se soslaya debido a la acción permanente pero constante de los mecanismos y representaciones propios del campo (autonomía científica, libertad de investigación, entre otras nociones ampliamente difundidas). Por el contrario, en momentos de crisis la relación entre ciencia y política 
se percibe con nitidez. Estos conflictos incluso han demostrado actuar como disparadores para la reflexión sobre las carencias en políticas de CyT durante los períodos cuando esta no se había puesto en cuestión. Particularmente, en la coyuntura analizada el foco estuvo puesto en los recortes y retrocesos sufridos por el sector desde 2015, pero también hubo lugar a reivindicaciones históricas y aún no resueltas y aún no resueltas del personal de CONICET en lo que hace al estatuto laboral de becarios y personal administrativo contratado.

Al decir de Echeverría $(2003,46)$, "las comunidades científicas siempre han procurado incidir en ámbitos políticos, tanto para obtener financiación para su actividad como para mostrar la utilidad social y política de sus investigaciones". También Elzinga y Jamison (1996) señalan la existencia de una cultura científica que disputa la política de la ciencia para influir en las políticas científicas. Sin embargo, como hemos visto, las acciones para incidir en los ámbitos políticos son múltiples. Los actores involucrados implementan diversas estrategias en función del contexto, los capitales a su disposición y las contingencias. En ocasiones, interpelan directamente a los actores relevantes del Estado, o bien pretenden extender una representación positiva de su actividad que influya en las decisiones de las elites gobernantes y sectores empresariales. Las prácticas y enunciados de CPCT pueden ser una forma de incidir en el ámbito político en este último sentido. Tienen la peculiaridad de que promueven una representación legitimadora de la CyT y sus agentes ante un conjunto amplio de la ciudadanía ajena a la comunidad científica.

Así, la CPCT puede entenderse parcialmente como una herramienta para captar la atención de la ciudadanía y de esta manera granjearse su apoyo. Pero esto simplifica en exceso las implicaciones de este tipo de comunicación. Se trata, más bien, de generar estrategias de reconocimiento y autorreconocimiento. Es decir, mediante la CPCT se pretende que los actores no científicos reconozcan la importancia de la ciencia y la tecnología, pero argumentamos que también son acciones que hacen que los propios agentes del campo científico se perciban a sí mismos como actores políticos aglutinados en una demanda común. No debemos olvidar que el campo científico es heterogéneo y organizar un accionar político en torno a una demanda colectiva requiere aglutinar agentes en posiciones dispares. En esta diversidad, hay actores con mayor propensión a implementar estrategias de visibilidad en la esfera pública y con intención de instalarlas como tácticas legítimas entre sus pares. Por medio de manifestaciones de CPCT para exhibir las problemáticas del sistema científico tecnológico se pretende, entonces, generar una doble interpelación tanto hacia el exterior como el interior del campo científico. Por un lado, generar un reconocimiento de la CyT en la ciudadanía y, por otro lado, movilizar a los agentes del campo más reacios a la acción política.

El reclamo básico de la comunidad científica, más allá del pedido por medidas concretas, es la consolidación de políticas científicas, aunque en el interior y el exterior del campo científico pueden existir disputas sobre sus características. 
Desde mediados del siglo pasado, la investigación científica y el desarrollo tecnológico requieren de grandes cantidades de recursos durante períodos prolongados para obtener resultados significativos. Esta condición es imposible de satisfacer sin la participación activa del Estado mediante políticas públicas orientadas al estímulo y fortalecimiento continuo de la $\mathrm{I}+\mathrm{D}$. Por consiguiente, las comunidades científicas pretenden influenciar a los órganos gubernamentales para instalar y fortalecer políticas públicas (Elzinga y Jamison, 1996) y en lo posible consolidar auténticas políticas de Estado (Hurtado, Bianchi y Lawler, 2017).

Sin embargo, exigir fondos del erario público para la práctica científica implica demostrar la relevancia de las investigaciones tanto a los tomadores de decisiones como a la ciudadanía. Esto obliga a los miembros del campo científico y sus aliados a desplegar diversas estrategias de comunicación orientadas a grupos no especializados en CyT. A tal fin, se deben recurrir a géneros textuales que no son los habitualmente utilizados para la comunicación endógena intra o inter disciplinar (Verón, 1998). La CPCT se torna un recurso para extender una representación positiva de la comunidad científica que no se puede lograr mediante la difusión de textos especializados. A través del recurso a una amplia gama de géneros textuales y estrategias comunicativas, se busca instalar una cultura ilustrada que legitime esfuerzos científicos cuyos efectos pueden no ser inmediatos, como en el caso de la ciencia básica.

Por otro lado, los comunicados emitidos por el Plenario de Directoras y Directores de Institutos del CONICET son una clara muestra del agotamiento de las vías de reclamo usuales para agentes ubicados en posiciones dominantes dentro del campo científico-académico. Los tres textos analizados remiten a la Reforma Universitaria de 1918 y a la Revolución de Mayo de 1810 en tanto acontecimientos sociales indiscutibles en el imaginario colectivo y asociados a valores como la libertad y la soberanía nacional, pero también a una manifestación de rebeldía en contra del orden establecido. Asimismo, la apelación a la Reforma del " 18 en particular traza una genealogía con un cierto ideal de "intelectual comprometido".

Así, tanto la CPCT como los comunicados se orientan al mismo objetivo de defender el sistema científico tecnológico, pero son géneros discursivos diferentes. En el análisis, reconocimos los lugares comunes en los comunicados y concluimos que es parte de la estrategia argumentativa. En efecto, la importancia de la CyT para el desarrollo y la mejora de la calidad de vida es parte del repertorio de tópicos de la hegemonía discursiva. Pueden variar estrategias para su estímulo e implementación, pero persiste un marco de consenso sobre la relevancia de la CyT suspendido en la doxa.

No obstante, la práctica científica y tecnológica suele no coincidir con la mayoría de las representaciones colectivas que circulan en sectores no especializados. Una CPCT crítica e integral puede exhibir la complejidad de la práctica de CyT $\mathrm{y}$ sus lógicas, incluso contraviniendo algunos de estos lugares comunes fuerte- 
mente instalados en el sentido común, sin dejar de resaltar sus virtudes. Es decir, tanto la comunicación política tradicional (por ejemplo, el Manifiesto y Llamamiento analizados) como la CPCT señalan la importancia y el valor social de la CyT. Pero la primera se apoya en la fuerza retórica que emana de las representaciones sociales del sentido común, mientras que la segunda tiene el potencial de desarticular la eficiencia retórica de esos argumentos apuntalados en la doxa. $¿ \mathrm{La}$ CPCT, entonces, es menos eficiente para generar argumentos a favor del sistema científico tecnológico? A nuestro parecer, la respuesta es no. La CPCT tiene el potencial de aportar nuevos y más sofisticados elementos para destacar el impacto de la CyT en nuestras vidas. Hay dos movimientos de comunicación, uno de clausura en los lugares comunes del discurso, el otro de apertura de la complejidad, ambos hacia una misma dirección: la defensa del sistema científico y tecnológico ante los vaivenes políticos.

Para finalizar, cabría plantear, como último y abierto interrogante, si la CPCT en sí misma también ha sido objeto de una defensa tan encarnizada como la propia CyT. Más allá de su utilidad como herramienta, ante la urgencia institucional y presupuestaria, parecería ocupar un lugar subsidiario en las manifestaciones: después de todo, si en el país no hay un sector científico-tecnológico, no habrá ciencia que comunicar. Sin embargo, en este trabajo hemos tratado de plantear la relevancia constitutiva de la CPCT respecto del campo científico, a través de las representaciones sobre la práctica científica y núcleos de sentido común que pone a circular en el espacio público.

En este sentido, ¿cómo pensar la relación entre CyT y CPCT en el marco de países periféricos? Podemos pensar un escenario donde el foco de los esfuerzos estatales estaría puesto en el sistema científico-tecnológico exclusivamente, cuyo desarrollo traccionaría la comunicación de esa ciencia. Por una suerte de efecto derrame, la CPCT sería un efecto secundario a la zaga de la expansión de la investigación y el desarrollo. La imagen que puede representar esta posibilidad es la de un cometa: la CyT, objeto de políticas científicas explícitas, arrastrando una cola dispersa y heterogénea de iniciativas de CPCT, movida por políticas implícitas, en términos de Herrera (2015 [1975]). Sin embargo, a nuestro juicio, esta forma de proceder es contraproducente para la consolidación de un sistema científico-tecnológico que fortalezca la autodeterminación y soberanía de nuestra región.

Otro abordaje implicaría poner a la CyT y su comunicación al mismo nivel, coordinadas por políticas integrales. Desde esta perspectiva, se reconoce el valor de la CPCT para construir un sentido común que respalde las iniciativas $\mathrm{Cy} \mathrm{T}$ y consolide a la potencia científica nacional dentro del imaginario social, de manera de trascender los momentos de retracción de las políticas públicas favorables al sector (Cortassa y Rosen, 2019). Más aún, la comunicación no debería ocupar un rol utilitario en las etapas de crisis del sistema científico-tecnológico, como hemos analizado. Al contrario, debe articularse activamente con la CyT en sus 
momentos de crecimiento y consolidación. La imagen que podemos asociar a este escenario alternativo es la de un sistema estelar binario en el cual dos estrellas se mantienen mutuamente en movimiento orbitando alrededor de un centro de masa. En este caso, concebimos en ese centro a un proyecto científico y tecnológico consecuente con un modelo de país soberano y desarrollado según sus propios términos y necesidades.

De lo expuesto se desprenden dos vías de acción y reflexión. En términos operativos, así como es urgente reformular y revisitar las políticas públicas en CyT en marco de la crisis, es imperativo repensar a la par y en la misma jerarquía de necesidades a la CPCT. En otras palabras, dar lugar a las políticas explícitas de CPCT en el seno de las políticas científicas. Por otra parte, en el ámbito académico, puede ser de utilidad una mayor profundización en la dimensión política de la CPCT y su concretización con las políticas públicas de CPCT. Es decir, su operatoria tanto en la política de la ciencia como en las políticas científicas. Como vimos, este abordaje permite, subsidiariamente, comprender mejor al mismo campo científico como agente político.

\section{Referencias bibliográficas}

Alcíbar, Miguel (2009). Comunicación pública de la tecnociencia: más allá de la difusión del conocimiento. Zer, 14(27), 165-188.

Alcíbar, Miguel (2015). Comunicación pública de la ciencia y la tecnología: una aproximación a su historia conceptual. Arbor, 191(773), a242. http://doi.org/10.3989/ arbor.2015.773n 3012

Bajtín, Mijail (2008). Estética de la creación verbal. Buenos Aires: Siglo XXI.

Bourdieu, Pierre (1994). El campo científico. REDES, 1(2), 131-160.

Céspedes, Lucía (2019). La revista argentina Ciencia Nueva (1970-1974), análisis de contenidos, recursos gráficos, publicidad y públicos. Perspectivas de la Comunicación, 12(1), 281-313.

Consejo Nacional De Investigaciones Científicas y Técnicas (2018). CONICET en cifras. Recuperado de https://cifras.conicet.gov.ar/publica/

Cortassa, Carina y Rosen, Cecilia (2019). Comunicación de las ciencias en Argentina: escenarios y prácticas de un campo en mutación. ArtefaCToS, 8(1), 61-81. http:// doi.org/10.14201/art2019816181

Diamand, Marcelo (1983). El péndulo argentino, ¿hasta cuándo? Buenos Aires: Centro de Estudios de la Realidad Argentina.

Echeverría, Javier (2003). La revolución tecnocientifica. Madrid: Fondo de Cultura Económica.

Elzinga, Aant y Jamison, Andrew (1996). El cambio de las agendas políticas en ciencia y tecnología. Zona Abierta, 75/76, 91-132. 
Feld, Adriana (2019). Science, Politics/Policy and the Cold War in Argentina: from Concepts to Institutional Models in the 1950s and '60s. Minerva, 57(4), 523547. https://doi.org/10.1007/s11024-019-09379-0

Herrera, Amilcar (2015 [1975]). Ciencia y politica en América Latina. Buenos Aires: Biblioteca Nacional.

Hilgartner, Stephen (1990). The Dominant View of Popularization: Conceptual Problems, Political Uses. Social Studies of Science, 20(3), 519-539.

Hurtado, Diego (2019). Proyectos de país en disputa: ¿qué hacer con la ciencia y la tecnología? Ciencia, Tecnología y Política, 2(2), 45-52.

Hurtado, Diego, Bianchi, Matías y Lawler, Diego (2017). Tecnología, políticas de Estado y modelo de país: el caso ARSAT, los satélites geoestacionarios versus "los cielos abiertos". Epistemología e Historia de la Ciencia, 2(1), 48-71.

Kreimer, Pablo (2006). ¿Dependientes o integrados? La ciencia latinoamericana y la nueva división internacional del trabajo. Nómadas, 24, 199-212.

Kreimer, Pablo (2011). La evaluación de la actividad científica: desde la indagación sociológica a la burocratización. Dilemas actuales. Propuesta educativa, 2(36), 5977.

Kreimer, Pablo, Levin, Luciano y Jensen, Pablo (2011). Popularization by Argentine researchers: the activities and motivations of CONICET scientists. Public Understanding of Science, 20(1), 37-47. https://doi.org/10.1177/0963662510383924

Mesa Coordinadora del Plenario Nacional de Directoras y Directores de Institutos del CONICET (2019a). Manifiesto de Córdoba 2019. Recuperado de https://cienciaenargentina.wixsite.com/mesacoordinadora

Mesa Coordinadora del Plenario Nacional de Directoras y Directores de Institutos del CONICET (2019b). Llamado a la ciudadanía: ;salvemos la ciencia argentina! Recuperado de https://cienciaenargentina.wixsite.com/mesacoordinadora

Mesa Coordinadora del Plenario Nacional de Directoras y Directores de Institutos del CONICET (2019c). A la ciudadanía y al conjunto de las fuerzas politicas argentinas: jsin ciencia no hay futuro! Recuperado de https://cienciaenargentina.wixsite. com/mesacoordinadora

Nocetti, Lidia, Palioff, Claudia y Della Torre, Virginia (2018). Conciencia de género. Políticas, números y algo más sobre el INTA. Buenos Aires: INTA Ediciones. Recuperado de: https://inta.gob.ar/sites/default/files/conciencia_de_genero.pdf

Red de Indicadores de Ciencia y Tecnología Iberoamericana e Interamericana (2018). El estado de la ciencia, principales indicadores de ciencia y tecnología iberoamericanos e interamericanos 2018. Buenos Aires: RICyT. Recuperado de http://www.ricyt. org/files/edlc_2018.pdf

Verón, Eliseo (1998). Entre la epistemología y la comunicación. CIC, Cuadernos de Información Cientifica, 4, 149-155. 